2005

\title{
Size-Dependent Properties Of Cdse Quantum Dots
}

\author{
S. Neeleshwar \\ C. L. Chen \\ C. B. Tsai \\ Y.Y.Chen \\ C. C. Chen \\ See next page for additional authors
}

Follow this and additional works at: https://researchrepository.wvu.edu/faculty_publications

\section{Digital Commons Citation}

Neeleshwar, S.; Chen, C. L.; Tsai, C. B.; Chen, Y. Y.; Chen, C. C.; Shyu, S. G.; and Seehra, M. S., "Size-Dependent Properties Of Cdse Quantum Dots" (2005). Faculty Scholarship. 676.

https://researchrepository.wvu.edu/faculty_publications/676 
Authors

S. Neeleshwar, C. L. Chen, C. B. Tsai, Y. Y. Chen, C. C. Chen, S. G. Shyu, and M. S. Seehra 


\title{
Size-dependent properties of CdSe quantum dots
}

\author{
S. Neeleshwar, C. L. Chen, C. B. Tsai, and Y. Y. Chen* \\ Institute of Physics, Academia Sinica, Taipei, Taiwan, Republic of China \\ C. C. Chen \\ Department of Chemistry, National Taiwan Normal University, and Institute of Atomic and Molecular Sciences, \\ Academia Sinica, Taipei, Taiwan, Republic of China \\ S. G. Shyu \\ Institute of Chemistry, Academia Sinica, Taipei, Taiwan, Republic of China \\ M. S. Seehra \\ Physics Department, West Virginia University, Morgantown, West Virginia 26506, USA \\ (Received 16 March 2005; revised manuscript received 27 April 2005; published 23 May 2005)
}

\begin{abstract}
Temperature dependences of the magnetic susceptibility $\chi$ and heat capacity $C_{p}$ of CdSe quantum dots with size $d=2.8,4.1$, and $5.6 \mathrm{~nm}$ are compared to those of bulk CdSe to determine the size-dependent effects. With decreasing size $d$, the following effects are observed: (i) room temperature optical absorption shows a blueshift of the band gap; (ii) room temperature x-ray diffraction show wurtzite structure but with smaller lattice constants; (iii) magnetic susceptibility changes from negative (diamagnetic) for the bulk to positive $\chi$ with magnitude increasing with decreasing $d$; and (iv) the Sommerfeld constant $\gamma$ determined from the $C_{p} / T$ vs $T^{2}$ data increases. Possible explanations for these size-dependent properties are presented.
\end{abstract}

DOI: 10.1103/PhysRevB.71.201307

PACS number(s): 75.75. $+\mathrm{a}, 65.80 .+\mathrm{n}$

\section{INTRODUCTION}

In recent years, properties of nanosized materials have generated a great deal of interest because of the science involved in these studies and technological applications of the quantum dots (QDs). As the physical dimensions of the particle approach to the nanometer scales, quantization and surface effects begin to play an important role, leading to drastic changes in measured properties. ${ }^{1}$ Among the semiconductor QD, studies have been reported for the II-IV (Ref. 2) and III-V (Ref. 3) materials, where a shift in the electronic transitions to higher energies accompanied by an increase of the oscillator strength with the decrease in the particles size were reported. Applications of the semiconductor QD have been reported for photovoltaics, ${ }^{4}$ light emitting diodes, ${ }^{5}$ lasers, ${ }^{6}$ and biological imagings. ${ }^{7}$ Other reports studied include optical spectroscopy, ${ }^{8}$ photoconductivity, ${ }^{9}$ and LO-phonon coupling. ${ }^{10}$

None of the studies listed above in semiconductor QD have focused on the effect of size on thermodynamic properties such as magnetic susceptibility $\chi$ and heat capacity $C_{p}$. Consequently in this paper we report detailed studies of the temperature dependence of $\chi$ and $C_{p}$ for CdSe quantum dots with size $d=2.8,4.1$, and $5.6 \mathrm{~nm}$ vis-a-vis bulk CdSe. Important size-dependent effects are observed, whose discussion and analysis are presented below.

\section{EXPERIMENTAL}

CdSe semiconductor quantum dots were prepared from the pyrolysis of dimethylcadmium and tri- $n$-octylphosphine selenide (TOPSe) in a hot coordinating solvent of tri- $n$-octylphosphine oxide (TOPO) using the procedure described previously. ${ }^{11}$ In this method, the surface of the CdSe quantum dot was passivated with TOPO molecules to avoid surface oxidation and aggregation. Different sizes of quantum dots were obtained by controlling its nucleation and growth process. For further size selection, size-selective precipitation can be carried out in a chloroform-methanol solvent system. Three sizes of quantum dots were prepared with $d=2.8,4.1$, and $5.6 \mathrm{~nm}$ with a standard deviation of $\sim 10 \%$ as determined by the high-resolution transmission electron microscopy (HRTEM); see inset of Fig. 1 for the 5.6-nm QD. Optical absorption spectra of CdSe quantum dots were obtained by a HP 8452 diode array spectrophotometer using

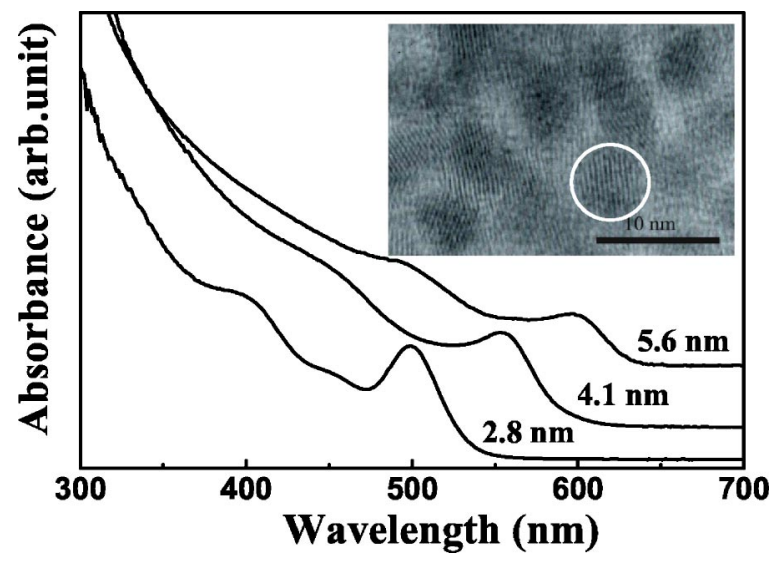

FIG. 1. Optical absorption spectra for $d=2.8-, 4.1-$, and 5.6-nm CdSe quantum dots dispersed in chloroform were taken at $300 \mathrm{~K}$. Inset: The HRTEM image of 5.6-nm CdSe quantum dots; an example particle marked by a circle is shown. 


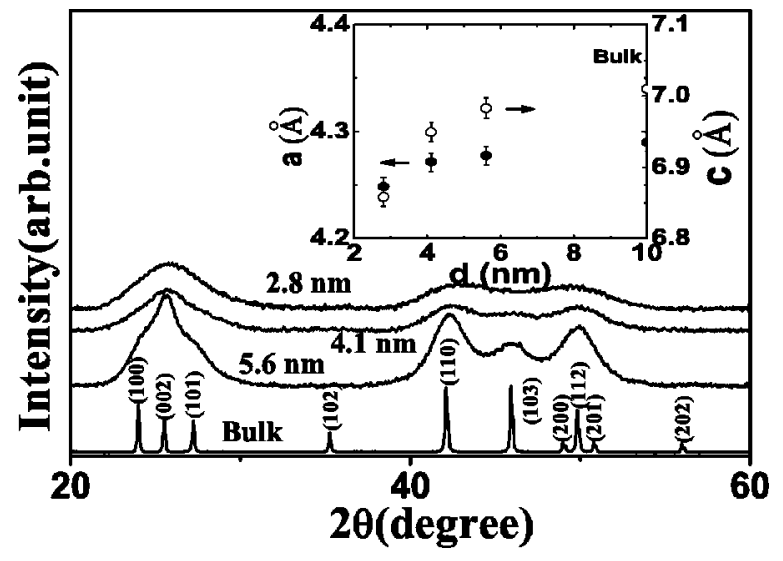

FIG. 2. X-ray diffraction patterns for the bulk and $d=2.8-, 4.1-$, and 5.6-nm quantum dots. Inset: The size dependence of lattice constants of $a$ and $c$ axes.

1-cm quartz cuvettes at room temperature as shown in Fig. 1. The blueshift of the absorption edge with the decreasing $d$ of quantum dots is consistent with an earlier report. ${ }^{12} \mathrm{X}$-ray diffraction (XRD) of the quantum dots (carried out with a 3-KW Philips diffractometer equipped with an array detector based on a real-time multiple strip) showed the wurtzite structure of the bulk CdSe but with the expected line broadening with decreasing $d$ (Fig. 2). In addition, there is a shrinkage of the lattice constants (the inset of Fig. 2), due to size effect, somewhat similar to that reported in the literature. ${ }^{13}$ No additional lines due to any impurity phase could be detected in the XRD spectra.

A calorimetric study was made in the range of 0.4 to 10 $\mathrm{K}$, using a thermal-relaxation microcalorimeter in a ${ }^{3} \mathrm{He}$ cryostat. ${ }^{14}$ Each milligram-sized sample was prepared by lightly pressing fine powders together. It was then attached with thermal-conducting $N$ grease to a sapphire disk, having two deposited thin films serving as heater and thermometer, respectively. The heat capacity of the sapphire disk and grease were measured separately, and used as addenda correction in data analysis. The relative precision and the absolute accuracy of the calorimeter were confirmed to be within $3 \%$ by measuring the copper standard. Magnetization measurements were performed as a function of temperature using the Quantum Design superconducting quantum interference device (SQUID) magnetometer in the range 2 to $300 \mathrm{~K}$. The magnetic susceptibility of straw and capsule were measured separately and subtracted from the data.

\section{RESULTS AND DISCUSSION}

The temperature dependence of magnetic susceptibility $\chi$ for CdSe QD with size $d=2.8,4.1$, and $5.6 \mathrm{~nm}$ and bulk $\mathrm{CdSe}$ is shown in Fig. 3. For the bulk CdSe, $\chi$ is diamagnetic and temperature independent with the magnitude $\approx-43 \times 10^{-6} \mathrm{emu} / \mathrm{mole}$ in good agreement with the earlier results. ${ }^{15}$ We report that for $\mathrm{QD}, \chi$ is positive and it has a strong temperature dependence, especially below $30 \mathrm{~K}$. Also the magnitude of $\chi$ is larger for the smaller particles at all temperatures, showing the effect of size on magnetism.

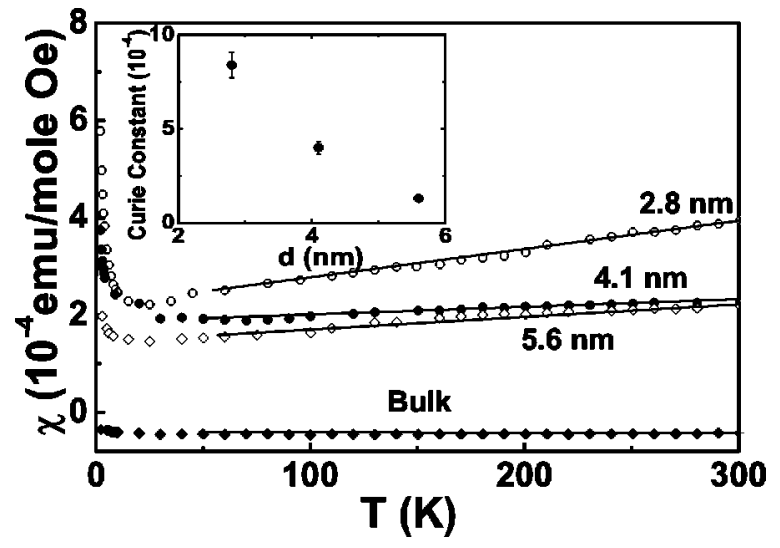

FIG. 3. The magnetic susceptibility as function of temperature for the bulk and $d=2.8-, 4.1-$, and 5.6-nm quantum dots; the lines are for eye's guide. Inset: The Curie constant vs $d$.

In general for pure semiconductors, $\chi=\chi_{l}+\chi_{f}+\chi_{i}$, where $\chi_{l}$ is the temperature-independent lattice contribution, $\chi_{f}$ is the free charge carrier (electrons and holes) contribution and $\chi_{i}$ is the contribution from bounded carriers and dangling bonds. For bulk CdSe, $\chi_{f}, \chi_{i} \ll \chi_{l}$, leading to magnetic susceptibility determined by $\chi_{l}$, which is usually negative (Fig. 3). ${ }^{15}$ Shaldin et al. ${ }^{16}$ have shown that in II-IV semiconductors, vacancies and interstitial can occur during the growth. For QD, such defects will be more prevalent as compared to bulk materials because of the increase in the relative surface area. Specific magnetic clusters created by the donoracceptor pairs can exhibit paramagnetic behavior. ${ }^{17}$ On the surfaces of semiconductors, the free dangling bond bears an electron spin by nature and can make semiconductor surfaces magnetic. These phenomena are expected to be more significant in QD. ${ }^{18}$

With these considerations in mind, we suggest that the low-temperature Curie tail in $\chi$ is most likely due to surface dangling bonds. These surface dangling bonds result from decreased coordination of the surface atoms of the QD. We have fitted the low-temperature data for $T<30 \mathrm{~K}$ to the modified Curie law: $\chi=\chi_{o}+C / T$, where $\chi_{o}$ is temperature independent contribution mainly from Pauli paramagnetism of $\chi_{f}$ mentioned above. The details will be discussed later. The fits are excellent with $C=1.33,4.0$, and 8.38 (in units of $10^{-4} \mathrm{emu} \mathrm{K} / \mathrm{mol}$ ) for $d=5.6,4.1$, and $2.8 \mathrm{~nm}$, respectively (inset to Fig. 3). This rapid increase in $C$ with a decrease in $d$ is due to increase in surface/volume ratio as $d$ decreases. Note that $C=N \mu^{2} / 3 k_{B}$ where $N$ is the number of dangling bonds/mol, each with effective magnetic moment $\mu$ and $k_{B}$ is the Boltzmann constant. If we assume spin $S=1 / 2$ with each dangling bond, leading to $\mu=1.73 \mu_{B}$, then $N=13.5 \times 10^{20} / \mathrm{mol}$ for $d=2.8 \mathrm{~nm}$, thus yielding the concentration of the dangling bonds $\approx 2000 \mathrm{ppm}$. For $d=4.1$ and $5.6 \mathrm{~nm}$, a similar calculation yields the concentration $\approx 1000$ and $300 \mathrm{ppm}$, respectively. It is noted that in amorphous $\mathrm{Si}$ and Ge, low-temperature magnetic susceptibility studies yielded similar concentration of spin density due to dangling bonds. ${ }^{19}$

The increase in $\chi$ with increasing temperature above $30 \mathrm{~K}$ seen for the QD in Fig. 3 is another interesting feature of our 


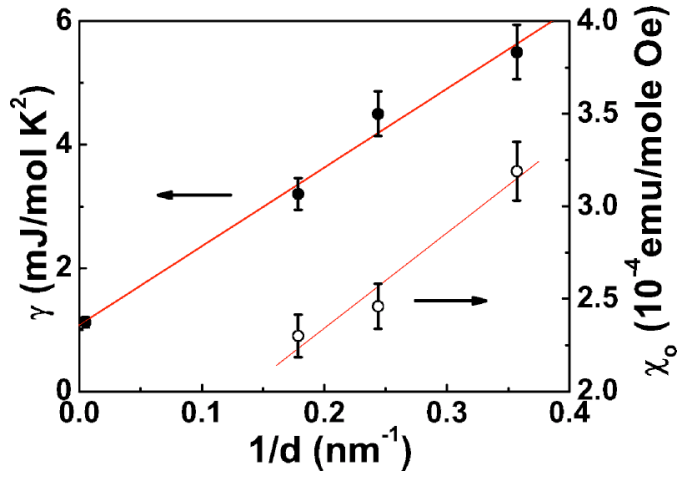

FIG. 4. The Sommerfield constant and $\chi_{o}$ (mainly from the contribution of free charge carrier) vs $1 / d(\sim$ surface/volume ratio), the lines are linear fits.

results. At the outset we note that a similar increase was reported by Burgardt and Seehra in semiconductor $\mathrm{FeS}_{2} \cdot{ }^{20} \mathrm{In}$ Fig. 3, both the magnitude and the slope increase with decrease in d. For $\mathrm{FeS}_{2}$ (Ref. 20) and amorphous $\mathrm{Si}$ and $\mathrm{Ge},{ }^{21}$ the positive $\chi$ and its temperature dependence at higher temperature were explained by the Van Vleck susceptibility

$$
\chi_{\mathrm{vv}}=2 N_{A} \mu_{B}^{2} \sum_{k} \frac{\left|\left\langle l\left|L_{Z}\right| k\right\rangle\right|^{2}}{E_{k}-E_{l}},
$$

where $N_{A}$ is the Avogadro's number and $L_{Z}$ is the $z$ component of the orbital angular momentum coupling the excited state $|k\rangle$ with energy $E_{k}$ with the ground state $|l\rangle$ with energy $\mathrm{E}_{l}$. For semiconductors, $E_{k}-E_{l} \geqslant E_{g}$ (energy gap). Note that $E_{g}$ usually decreases with increase in temperature ${ }^{2,22}$ and in CdS nanoclusters, a much steeper temperature dependence with decreasing particle size is observed. Assuming similar results are valid for $\mathrm{CdSe} \mathrm{QD}$, it then explains why $\chi$ increases with increasing temperature, and increasing slope with decreasing $d$, as observed in Fig. 3. To estimate $\chi_{\mathrm{vv}}$ from Eq. (1), if we approximate the sum over all the states by $1 / E_{g}$ assuming the matrix elements to be unity, $\chi_{\mathrm{vv}}=0.3 \times 10^{-4} \mathrm{emu} / \mathrm{mol}$ Oe is obtained for $E_{g} \simeq 1.75 \mathrm{eV}$ valid for CdSe QD. This estimate of $\chi_{\mathrm{vv}}$ is about a factor of three times smaller than the enhancement of $\chi$ observed for QD. This confirms that $\chi_{o}$ is mainly contributed by $\chi_{f}$ as proposed earlier. This issue requires further investigation.

Since the surface free charge carriers (which gives $\chi_{f}$ ) are easily formed in QDs, ${ }^{13}$ the increase in the number of free charge carriers with surface for smaller particles is expected to vary as $1 / d$. In Fig. $4 \chi_{o}$ vs $1 / d$ shows linear dependence. The fact that there are systematic changes, in both the magnitudes and temperature dependence of $\chi$, with the particle size $d$ suggests that $\chi$ is dominated by the size effect and surface effects rather than any impurity.

To further examine the consequences of the size effect, measurements of specific heat of bulk $\mathrm{CdSe}$ and quantum dots with size $d=2.8,4.1$, and $5.6 \mathrm{~nm}$ were carried out for $T=0.3-40 \mathrm{~K}$. The temperature dependence of specific heat for the bulk CdSe and quantum dots, plotted as $C / T$ vs $T^{2}$ is shown in Fig. 5. The heat capacity of the bulk is in good agreement with earlier report. ${ }^{23}$ The enhancement of specific heat of quantum dots as the evolution of size is clearly re-

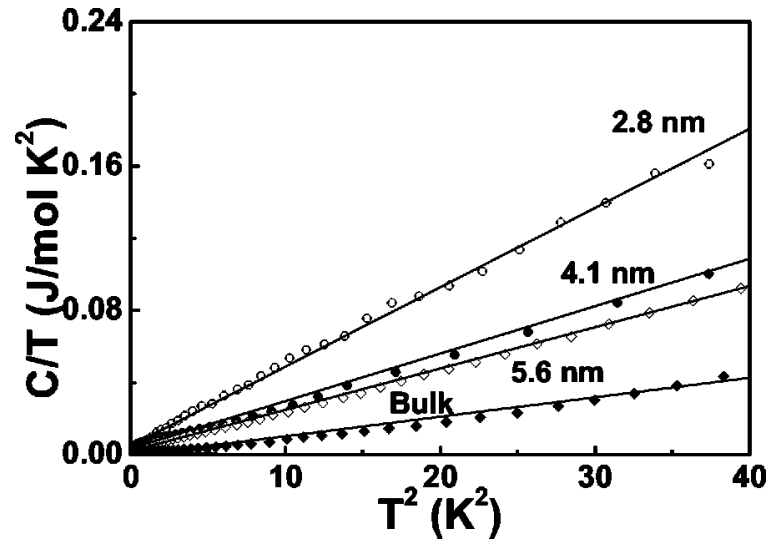

FIG. 5. The specific heat, plotted as $C / T$ vs $T^{2}$ for the bulk and $d=2.8-, 4.1-$, and 5.6-nm quantum dots; the lines are linear fits.

vealed. In general, the specific heat of a material can be represented by the summation of contributions of conduction electrons $C_{\mathrm{el}}=\gamma \mathrm{T}$, lattice phonon $C_{\mathrm{ph}}$ and magnetic correlations $C_{\mathrm{mag}}$. The value of the Sommerfeld constant $\gamma$ obtained from the intercept of the linear fits gradually increases from $1.12 \mathrm{~mJ} / \mathrm{mole} \mathrm{K}^{2}$ for the bulk to $5.50 \mathrm{~mJ} / \mathrm{mole} \mathrm{K}^{2}$ for 2.8 $\mathrm{nm}$ with decreasing $d$. The relation of $\gamma$ and the density of states of conduction electrons $N\left(\varepsilon_{F}\right)$ can be represented by

$$
\gamma=\frac{1}{3} \pi^{2} k_{B}^{2} N\left(\varepsilon_{F}\right)
$$

where $k_{B}$ is Boltzmann constant. The value of $\gamma$ increases with decreasing $d$ and indicates an enhancement of density of states of conduction electrons $N\left(\varepsilon_{F}\right)$ in quantum dots. The value of $\gamma$ is approximately linear proportional to $1 / d$, implying the correlation of the density of states of conduction electrons $N\left(\varepsilon_{F}\right)$ with the surface of quantum dot (Fig. 4). Since $\chi_{f}$ is also proportional to $N\left(\varepsilon_{F}\right)$, the similar variations of $\gamma$ and $\chi_{o}$ are understandable. It is noted that quantum dots have an enormous surface-to-volume ratio; consequently, the free charge from delocalized electrons of dangling bonds and defects on surface will have more contribution to magnetic susceptibility $\chi_{o}$ and heat capacity $\gamma$ as well. For quantum dots, the lattice phonons $C_{\mathrm{ph}}$ can be calculated by the theoretical model for a small particle represented by the following equation: ${ }^{24}$

$$
C_{\mathrm{ph}}=V_{m} \sum_{l, s} \frac{3(2 l+1) k_{B} x^{2} e^{x}}{4 \pi R^{3}\left(e^{x}-1\right)^{2}}, \quad \text { with } x=\frac{\hbar c a^{\prime}{ }_{l, s}}{R T} .
$$

Here $V_{m}$ is the molar volume, $R$ denotes the particle radius, $a_{l, s}^{\prime}$, is the sth zero of the derivative of the $l$ th spherical Bessel function, and $c$ is the effective sound velocity. The number of atoms $N_{o}$ in quantum dots with $d=2.8,4.1$, and $5.6 \mathrm{~nm}$ is estimated to be about 500,1200 , and 2200 , respectively. We use the constraint $\Sigma_{l}^{l \max }(2 l+1)=N_{o}$ and subtract the contribution to the heat capacity from free charge carriers $C_{\mathrm{el}}=\gamma T$. The remaining heat capacity yields $C_{\mathrm{ph}}$ from which $c=795,895,915 \mathrm{~m} / \mathrm{s}$ with Debye temperature $\Theta=61,68,70$ $\mathrm{K}$ are obtained for $d=2.8,4.1$, and $5.6 \mathrm{~nm}$, respectively. 
Compared to $\Theta=139 \mathrm{~K}$ for bulk $\mathrm{CdSe}, \Theta$ for the quantum dots are really half, an anticipated result from lattice softening with decreasing $d .{ }^{24}$

\section{CONCLUSION}

The optical absorption spectra show a blueshift in the CdSe quantum dot. X-ray diffraction confirmed that QDs have the same wurtzite crystal structure as the bulk but with smaller lattice constants. The low-temperature magnetic susceptibility studies reveal the increase of spin density of dangling bonds with decreasing size. The magnetic susceptibility $\chi_{o}$ and Sommerfeld constant $\gamma$ increases linearly with surface-to-volume ratio, giving the evidence of free charge carriers on the surface of CdSe quantum dot. The systematic changes in the magnitudes of $\chi$ and $\gamma$ with the size $d$ suggest the role of quantum size effect and surface effects rather than any impurity in CdSe quantum dots.

\section{ACKNOWLEDGMENT}

This work was supported by the National Research Council of the Republic of China under Grant No. NSC 93-2112M-001-022.
*Electronic address: cheny2@phys.sinica.edu.tw

${ }^{1}$ A. P. Alivisatos, Science 271, 933 (1996).

${ }^{2}$ T. Vossmeyer, L. Katsikas, M. Giersig, I. G. Popovic, K. Diesner, A. Chemseddine, A. Eychmüller, and H. Weller, J. Phys. Chem. 98, 7665 (1994).

${ }^{3}$ A. A. Guzelian, U. Banin, A. V. Kadavanich, X. Peng, and A. P. Alivisatos, Appl. Phys. Lett. 69, 1432 (1996).

${ }^{4}$ R. P. Raffaelle, S. L. Castro, A. F. Hepp, and S. G. Bailey, Prog. Photovoltaics 10, 433 (2002).

${ }^{5}$ S. Coe, W. K. Woo, M. G. Bawendi, and V. Bulovic, Nature (London) 420, 800 (2002).

${ }^{6}$ V. I. Klimov, A. A. Mikhailovsky, S. Xu, A. Malko, J. A. Hollingsworth, C. A. Leatherdale, H. J. Eisler, and M. G. Bawendi, Science 290, 314 (2000).

${ }^{7}$ M. Bruchez, Jr., M. Moronne, P. Gin, S. Weiss, and A. P. Alivisatos, Science 281, 2013 (1998).

${ }^{8}$ M. Nirmal, C. B. Murray, and M. G. Bawendi, Phys. Rev. B 50, 2293 (1994).

${ }^{9}$ M. C. Beard, G. M. Turner, and C. A. Schmuttenmaer, Nano Lett. 2, 983 (2002).

${ }^{10}$ M. Nirmal, C. B. Murray, D. J. Norris, and M. G. Bawendi, Z. Phys. D: At., Mol. Clusters 26, 361 (1993).

${ }^{11}$ C. B. Murray, D. J. Norris, and M. G. Bawendi, J. Am. Chem.
Soc. 115, 8706 (1993)

${ }^{12}$ C. D. Dushkin, S. Saita, K. Yoshie, and Y. Yamaguchi, Adv. Colloid Interface Sci. 88, 37 (2000).

${ }^{13}$ C. B. Murray, C. R. Kagan, and M. G. Bawendi, Annu. Rev. Mater. Sci. 30, 545 (2000).

${ }^{14}$ Y. Y. Chen, Y. D. Yao, S. S. Hsiao, S. U. Jen, B. T. Lin, H. M. Lin, and C. Y. Tung, Phys. Rev. B 52, 9364 (1995).

${ }^{15}$ D. J. Chadi, R. M. White, and W. A. Harrison, Phys. Rev. Lett. 35, 1372 (1975).

${ }^{16}$ Y. V. Shaldin, I. Warchulska, M. K. Rabadanov, and V. K. Komar, Semiconductors 38, 288 (2004).

${ }^{17}$ J. van Wieringen, Philips Tech. Rev. 19, 301 (1957/1958).

${ }^{18}$ Takanori Suzuki, V. Venkataramanan, and Masakazu Aono, RIKEN Rev. 37, 9 (2001).

${ }^{19}$ S. J. Hudgens, Phys. Rev. B 14, 1547 (1976).

${ }^{20}$ P. Burgardt and M. S. Seehra, Solid State Commun. 22, 153 (1977).

${ }^{21}$ S. J. Hudgens, Phys. Rev. B 7, 2481 (1973).

${ }^{22}$ M. S. Seehra and S. S. Seehra, Phys. Rev. B 19, 6620 (1979).

${ }^{23}$ A. Twardowski, H. J. M. Swagten, and W. J. M. de Jonge, Phys. Rev. B 42, 2455 (1990).

${ }^{24}$ G. H. Comsa, D. Heitkamp, and H. S. Räde, Solid State Commun. 24, 547 (1977). 